\title{
The role of environmental stress and DNA methylation in the longitudinal course of bipolar disorder
}

\author{
Ashley L. Comes ${ }^{1,2^{*}} \mathbb{0}$, Darina Czamara ${ }^{3}$, Kristina Adorjan ${ }^{1,4}$, Heike Anderson-Schmidt1,5, Till F. M. Andlauer ${ }^{3,6}$, \\ Monika Budde ${ }^{1}$, Katrin Gade ${ }^{5}$, Maria Hake ${ }^{1}$, Janos L. Kalman 1,2,4, Sergi Papiol ${ }^{1,4}$, Daniela Reich-Erkelenz ${ }^{1}$, \\ Farah Klöhn-Saghatolislam 1,4, Sabrina K. Schaupp ${ }^{1}$, Eva C. Schulte ${ }^{1,4}$, Fanny Senner ${ }^{1,4}$, Georg Juckel ${ }^{7}$, \\ Max Schmauß ${ }^{8}$, Jörg Zimmermannn', Jens Reimer ${ }^{10}$, Eva Reininghaus ${ }^{11}$, lon-George Anghelescu ${ }^{12}$, \\ Carsten Konrad ${ }^{13}$, Andreas Thiel ${ }^{13}$, Christian Figge ${ }^{14}$, Martin von Hagen ${ }^{15}$, Manfred Koller ${ }^{16}$, \\ Detlef E. Dietrich ${ }^{17,18,19}$, Sebastian Stierl ${ }^{20}$, Harald Scherk ${ }^{21}$, Stephanie H. Witt ${ }^{22}$, Sugirthan Sivalingam ${ }^{23}$, \\ Franziska Degenhardt ${ }^{23}$, Andreas J. Forstner ${ }^{23,24,25,26}$, Marcella Rietschel ${ }^{22}$, Markus M. Nöthen ${ }^{23}$, \\ Jens Wiltfang 5,27,28, Peter Falkai ${ }^{4}$, Thomas G. Schulze ${ }^{1,4+}$ and Urs Heilbronner ${ }^{1+}$
}

\begin{abstract}
Background: Stressful life events influence the course of affective disorders, however, the mechanisms by which they bring about phenotypic change are not entirely known.

Methods: We explored the role of DNA methylation in response to recent stressful life events in a cohort of bipolar patients from the longitudinal PsyCourse study $(n=96)$. Peripheral blood DNA methylomes were profiled at two time points for over 850,000 methylation sites. The association between impact ratings of stressful life events and DNA methylation was assessed, first by interrogating methylation sites in the vicinity of candidate genes previously implicated in the stress response and, second, by conducting an exploratory epigenome-wide association analysis. Third, the association between epigenetic aging and change in stress and symptom measures over time was investigated.

Results: Investigation of methylation signatures over time revealed just over half of the $\mathrm{CpG}$ sites tested had an absolute difference in methylation of at least 1\% over a 1-year period. Although not a single CpG site withstood correction for multiple testing, methylation at one site (cg15212455) was suggestively associated with stressful life events $\left(p<1.0 \times 10^{-5}\right)$. Epigenetic aging over a 1 -year period was not associated with changes in stress or symptom measures.

Conclusions: To the best of our knowledge, our study is the first to investigate epigenome-wide methylation across time in bipolar patients and in relation to recent, non-traumatic stressful life events. Limited and inconclusive evidence warrants future longitudinal investigations in larger samples of well-characterized bipolar patients to give a complete picture regarding the role of DNA methylation in the course of bipolar disorder.
\end{abstract}

Keywords: DNA methylation, Bipolar disorder, Stressful life events, Longitudinal, Epigenomics, Epigenetic aging

\footnotetext{
*Correspondence: Ashley.Comes@med.uni-muenchen.de

†Thomas G. Schulze and Urs Heilbronner contributed equally to this work ${ }^{1}$ Institute of Psychiatric Phenomics and Genomics, University Hospital, LMU Munich, Nussbaumstrasse 7, 80336 Munich, Germany

Full list of author information is available at the end of the article
}

\section{Background}

Bipolar disorder (BD) remains an interesting candidate for neurobiological analyses owing to its heterogenous presentation and both genetic and environmental risk factors (Ludwig and Dwivedi 2016). While genome-wide 
association studies (GWAS) in BD have identified dozens of associated variants, they have explained only a small fraction of overall disease liability (Stahl et al. 2019). Therefore, the last decade has seen a shift towards investigating the complex interplay between genetic and environmental risk factors (Sharma et al. 2016). Advances in technologies have supported high-throughput investigations of biological markers representative of environmental modulation of the genome. These biomarkers hold promise for stratifying symptom-based phenotypes and assessing the prognosis of individual patients (Kobeissy et al. 2012). Moreover, these biomarkers could contribute to a more accurate multi-level diagnostic framework which relies on biological measures to supplement clinical ratings of symptoms (Meana and Mollinedo-Gajate 2017).

$\mathrm{BD}$ is a chronic, disabling, and severe mental illness characterized by recurrent depressive and manic episodes, somatic and psychiatric comorbidities, and functional impairments (Goodwin and Jamison 2007). Considering the high global burden and lifetime prevalence of bipolar spectrum disorders, estimated at approximately 2.4\% (Rowland and Marwaha 2018), there is a need to better understand the factors affecting its onset and course. The significance of environment, especially childhood trauma and stressful life events on the trajectories of affective disorders, including vulnerability, onset, relapse and occurrence, has been well established (Aldinger and Schulze 2017; Lex et al. 2017; Johnson 2005; Alloy et al. 2005; Paykel 2003). However, little is known about the mechanisms involved in the consequences of such life events.

Recently, emphasis has been placed on the potential role of epigenetic variation in the etiopathogenesis of $\mathrm{BD}$ ( $\mathrm{Li}$ et al. 2015). Epigenetics is an adaptive mechanism which can modulate the stress response through subtle gene expression modifications (Aas et al. 2016). In particular, DNA methylation (DNAm), the addition of a methyl group to DNA, primarily at cytosine-guanine dinucleotides (CpG), may pose a "mechanism by which life-experiences become 'embedded' in the genome" (Marzi et al. 2018).

Increasing evidence from both animal and human data supports the epigenetic programming of genes in response to trauma and chronic stress. Consistent findings have linked prenatal (Monk et al. 2012; Weaver et al. 2004) and early-life adversities to epigenetic modifications of genes, especially those involved in the hypothalamic-pituitary-adrenal (HPA) axis (Kular and Kular 2018; McGowan et al. 2009; Vinkers et al. 2015; Jaworska-Andryszewska and Rybakowski 2019). While several studies have shown methylation changes associated with trauma during the adult period, few studies have investigated non-traumatic chronic stress (Matosin et al. 2017) or acute stressful life events. Candidate gene approaches in the general population have reported differential methylation of CpGs in the vicinity of SLC6A4 (Alasaari et al. 2012), $T H$ (Myaki et al. 2015), and BDNF (Song et al. 2014) in association with sustained workrelated stress. One study, which examined LINE-1 as a proxy for global methylation, found no signification associations with chronic lifestyle stress (Duman and Canli 2015). To the best of our knowledge, not a single study has explored epigenome-wide signatures of DNAm in relation to acute, non-traumatic stress in humans. With regards to $\mathrm{BD}$, studies have investigated methylation differences as both trait and state markers of the disorder in several promoter regions including SLC6A4, PPIEL31, BDNF, HCG9, KCNQ3, 5HTR1A and GPR24 (Ludwig and Dwivedi 2016; Fries et al. 2016; Pishva et al. 2014). Interestingly, evidence supports altered DNAm profiles for high-risk affected and even unaffected offspring of individuals with BD in comparison to low risk controls. Moreover, there seems to be a unique rate of change in DNAm over time for high risk individuals (Duffy et al. 2019). However, despite findings of differential epigenetic profiles, results have been inconsistent and there remains a need for genome-wide methylation studies, especially ones longitudinal in design.

This study aims to gain a better understanding of the role of epigenetic modifications, specifically DNAm, in relation to stress during the course of $\mathrm{BD}$. Using repeated measures over a 1-year period, we explored the relationship between DNAm and stressful life events in chronic BD patients. We took a two-pronged approach, first by interrogating CpGs in the vicinity of candidate genes previously implicated in the stress response and, second, by conducting an exploratory epigenome-wide analysis. Furthermore, we determined whether changes in symptom and stress measures over time were associated with a DNAm-based age estimate and epigenetic aging.

\section{Methods \\ Study sample}

The study was conducted using data from the longitudinal PsyCourse cohort. PsyCourse has been described in detail (Budde et al. 2019). Briefly, PsyCourse is a multisite, naturalistic study, based in the German and Austrian population. Psychopathology, pharmacological treatment, childhood trauma and current stressful life events were among other variables assessed at each of four visits (6-month intervals). Likewise, peripheral blood samples were collected at each visit, paving the way for a detailed analysis of the longitudinal correlation between disease status and peripheral biomarkers. For the purpose of this study, a subset of PsyCourse participants $(n=96)$ was 
selected according to a DSM-IV diagnosis (American Psychiatric Association 2002) of type I or II BD, availability of genotype data and biomaterial, and completed childhood trauma and stressful life events questionnaires. Demographic and clinical characteristics of these patients are reported in Table 1. The study was approved by the local ethics committee for each study center and was carried out following the rules of the Declaration of Helsinki. All individuals provided written informed consent.

\section{Measures}

\section{Stressful life events}

Current stressful life events were assessed with the Life Events Questionnaire (LEQ), a 79-item self-report instrument that has been described in detail (Norbeck 1984; Sarason et al. 1978). The LEQ covers a wide range of stressor exposure related to health, work, school, residence, love and marriage, family and friends, parenting, the personal sphere or social environment, finances, crime and legal matters. At each visit, participants reported whether they experienced any of the listed events in the last 6 months. When the patient experienced a specific event, they rated: (1) the nature of the

Table 1 Sample demographic and clinical characteristics

\begin{tabular}{|c|c|c|c|}
\hline & $\begin{array}{l}\text { Baseline } \\
(n=96)\end{array}$ & $\begin{array}{l}\text { 1-year follow-up } \\
(n=95)\end{array}$ & $p$-value \\
\hline \multicolumn{4}{|l|}{ Sex } \\
\hline Female & 50 & 50 & \\
\hline Age, mean $\pm S D$ & $45.2 \pm 12.4$ & $46.17 \pm 12.4$ & \\
\hline $\begin{array}{l}\text { Duration of illness, } \\
\text { mean } \pm S D\end{array}$ & $13.52 \pm 11.8$ & $14.66 \pm 11.8$ & \\
\hline \multicolumn{4}{|l|}{ DSM-IV diagnosis } \\
\hline BD-I & 79 & 78 & \\
\hline BD-II & 17 & 17 & \\
\hline \multicolumn{4}{|l|}{ Medication } \\
\hline Combo therapy & 81 & 75 & \\
\hline Monotherapy & 14 & 16 & \\
\hline No meds & 1 & 4 & \\
\hline Childhood trauma (yes) & 48 & 48 & \\
\hline \multicolumn{4}{|l|}{ LEQ scores, mean $\pm S D$} \\
\hline Bad events & $10.2 \pm 13.8$ & $6.3 \pm 6.6$ & $0.004^{b}$ \\
\hline Good events & $9.7 \pm 10.2$ & $8.4 \pm 7.6$ & $0.191^{b}$ \\
\hline Total events & $19.9 \pm 18.4$ & $14.1 \pm 10.7$ & $0.001^{b}$ \\
\hline \multicolumn{4}{|l|}{ Symptom ratings } \\
\hline $\mathrm{GAF}$, mean $\pm \mathrm{SD}$ & $61.5 \pm 12.6$ & $65.8 \pm 12.4$ & $0.032^{\mathrm{a}}$ \\
\hline YMRS sum, mean \pm SD & $3.9 \pm 5.8$ & $2.4 \pm 3.7$ & $0.216^{\mathrm{b}}$ \\
\hline$I D S-C_{30}$, mean $\pm S D$ & $13.7 \pm 11.0$ & $10.6 \pm 9.7$ & $0.124^{b}$ \\
\hline PANSS sum, mean $\pm S D$ & $42.8 \pm 11.8$ & $39.2 \pm 9.6$ & $0.063^{b}$ \\
\hline
\end{tabular}

\footnotetext{
a Paired sample t-test

b Wilcoxon signed rank test
}

event (good/bad) and (2) the impact of the event on his/ her life (0-3). At each time point, adverse life events were summed to yield a stress score that reflects the impact ratings of all "bad" events. The same was done for the impact ratings of "good" events. A total score was also summed including impact ratings of both "bad" and "good" events. These three LEQ scores were used as outcome measures in our association analyses.

\section{Childhood trauma}

The Childhood Trauma Screener (CTS) is a German, short version of the Childhood Trauma Questionnaire (Bernstein et al. 1997, 2003; Grabe et al. 2012). The screener includes five questions to assess sexual, physical and emotional abuse, as well as emotional and physical neglect. Validated threshold values (Glaesmer et al. 2013) were used to transform ratings for each item into a dichotomous scale in order to identify individuals with reported childhood trauma (yes/no). Details on reported childhood trauma and thresholds used can be found in Additional file 1: Table S1.

\section{Symptom ratings}

The Positive and Negative Syndrome Scale (PANSS) was used as a measure of psychopathology at the time of testing (Kay et al. 1987). A continuous total score of the three subscales, i.e. positive, negative, and general symptoms was used. The Global Assessment of Functioning (GAF) score was used as a measure of psychosocial functioning (Luborsky 1962; Endicott et al. 1976). The Young Mania Rating Scale (YMRS) was used as a measure of manic symptoms in the last $48 \mathrm{~h}$ (Young et al. 1978). Lastly, the Inventory of Depressive Symptomatology (IDS- $\mathrm{C}_{30}$ ), a 30-item rating scale, was used to assess the severity of depressive symptoms (Trivedi et al. 2004).

\section{Analysis of DNA methylation DNA samples}

Genomic DNA was extracted from whole blood using the PerkinElmer Chemagen Kit (chemagic DNA Blood10k prefilling VD120419.che) and all samples were subsequently stored in a Hamilton Bios $\mathrm{M}$ system at $-80{ }^{\circ} \mathrm{C}$. DNA quality was assessed using the QIAxcl $^{\circledR}$ system. DNA samples from baseline and 1-year follow-up visits were used to obtain methylation data. Prior to downstream analyses, potential population stratification was evaluated, and an initial step to remove European population outliers was taken (Budde et al. 2019). Thus, our sample consists of an ethnically homogenous population of Caucasians of European descent. 


\section{Illumina EPIC chip processing}

Bisulfide conversion of DNA and processing of methylation arrays was accomplished in collaboration with the Institute of Human Genetics, University of Bonn, Germany. Whole-blood genomic DNA diluted with water $(50 \mathrm{ng} / \mu \mathrm{l})$ was treated with sodium bisulfite using the EpiTect $^{\circledR}$ Bisulfite Kit from QIAGEN ${ }^{\circledR}$ following the manufacturer's protocol. DNAm was assessed using the Illumina Infinium Human MethylationEPIC BeadChip array (Illumina Inc., San Diego, CA, USA) according to the manufacturer's instructions. To minimize batch effects during DNAm measurement, an algorithm for sample randomization was used for positioning samples onto 96-well plates according to exposures of interest and confounding variables (see Additional file 1).

\section{Quality control and normalization Quality control}

The Bioconductor $\mathrm{R}$ package minfi was used to read raw intensity data files (.idat files) into $\mathrm{R}$ and for the subsequent quality control and normalization of methylation data (Aryee et al. 2014). Concordance between methylation-predicted and reported sex was confirmed. Filtering of poor-performing samples and probes was performed (see Additional file 1: Table S2). Probes with low detection $p$-values $(>0.05$ in $>10 \%$ of samples) were excluded. Using the function dropLociWithSnps(), SNPs inside the probe body and at the nucleotide extension were removed according to a minor allele frequency $\geq 5 \%$ based on dbSNP. To prevent a possible gender effect, $\mathrm{X}$ and $\mathrm{Y}$ chromosomes were removed. According to a list previously published (Chen et al. 2013), non-specific probes i.e. probes on the EPIC array that co-hybridize to alternate genomic sequences, were removed. Lastly, probes with a bead count $<3$ were removed.

\section{Normalization}

Data were normalized using functional normalization (FunNorm), an extension of quantile normalization. FunNorm uses internal control probes present on the array to infer between-array technical variation, by default using the first two principal components of the control probes (Fortin et al. 2014). Density plots were used to evaluate the distribution of $M$-values before and after functional normalization (see Additional file 1: Fig. S1).

Technical batch effects were then identified using linear regressions to inspect the association of principal components of the methylation values with possible technical batches. Additionally, the R package shinyM$e t h y l$ was used for visual inspection of principle component analysis (PCA) plots. Identified batch effects (i.e., array and slide) were removed using the Empirical Bayes' method ComBat (Johnson et al. 2007). Batch corrected
$M$-values after ComBat were used for downstream analyses (see Additional file 1: Fig. S2). According to inspection of PCA plots, a single sample remained an outlier after batch correction and was excluded.

\section{Confounders}

Considering cell-type composition is a confounding factor in epigenome-wide association studies (EWAS), the minfi function estimateCellcounts() was used to estimate the cell type composition for our samples. This function uses a modified version of the Houseman algorithm to obtain a cell counts vector for the six cell-types (i.e., CD4T, CD8T, NK, B cells, monocytes, and granulocytes) for each sample (Houseman et al. 2012).

Active smoking is another established modifier of DNA methylation (Lee and Pausova 2013). Methylation-based smoking scores were calculated based on the methylation profile of the $187 \mathrm{CpG}$ sites identified in Zeilinger et al. (2013). First, raw beta values were normalized using the Teschendorff et al. beta-mixture quantile dilation (BMIQ) strategy (Teschendorff et al. 2013). Adjusted beta-values were then used for calculation of methylation-based smoking scores using methods previously described (Elliott et al. 2014). The correlation between self-reported number of cigarettes smoked yearly and methylation-based smoking scores was assessed (Spearman's $\rho=0.64 ; p<0.001$ ).

To rule out possible confounding effects of medication, 5 samples were excluded in sensitivity analyses. These samples were participants who were not taking psychotropic drugs at the time of testing. All other participants were taking at least one (monotherapy) or a combination (combo therapy) of the following (1) antidepressants, (2) antipsychotics, (3) mood stabilizers, (4) tranquilizers, or (5) other psychiatric medications.

\section{Statistical analyses}

All statistical analyses were performed in $\mathrm{R}$ version 3.4.4 (http://www.r-project.org/) (R Core Team 2014).

\section{Change in methylation over time}

The general "stability" of methylation over time was investigated. First, the absolute change in methylation $\beta$-values between baseline and 1-year follow-up visits were calculated across all CpG sites. To determine whether differential methylation between visits remained significant after adjusting for known confounders, the package lme4 (Bates et al. 2015) was used to fit a linear mixed-effects model (LMM) with the dependent variable " $M$-value" and the independent variable "time", adjusting for age, sex, DNAm smoking scores, and cell composition estimates. Patient ID was included as the random effect term. 


\section{Candidate gene analysis}

The association between LEQ scores and the interaction between CT and total LEQ scores with DNAm was assessed via LMMs, adjusting for covariates as described above. We interrogated DNAm in the vicinity of genes previously implicated in the HPA-axis (i.e. $B D N F, F K B P 5, I L 6, S L C 6 A 4$, and OXTR). All probes on the EPIC array annotated to each of these five genes were identified. The number of probes per gene ranged from 22 to 124 . We corrected for multiple testing on a gene-level by applying the false discovery (FDR) correction (Benjamini and Hochberg 1995) per gene, with FDR-corrected $p$-values $\leq 0.05$ deemed significant. Afterwards, Bonferroni-correction was used to correct overall for the number of candidate-genes tested.

\section{Exploratory EWAS}

An exploratory EWAS was conducted. As a means of noise reduction, the top $10 \%$ of the most variable CpGs of the normalized, batch corrected $M$-values were extracted according to median absolute deviation (MAD) scores i.e. the median of the absolute deviations from the data's median. Associations between the most variable sites and LEQ scores and the interaction between childhood trauma and total LEQ scores were then tested using LMMs, adjusting for covariates as described above.

\section{Epigenetic aging}

DNAm-based age prediction was performed using the Horvath age estimation algorithm (Horvath 2013) with a freely available online tool (https://dnamage.genetics. ucla.edu/home) which predicts DNAm-age based on the methylation of $353 \mathrm{CpGs}$ using an elastic net penalized regression model. The difference between the estimated epigenetic age and chronological age $(\Delta$ age $)$ and a measure of epigenetic age acceleration (AA), i.e., the residual from regressing DNAm age on chronological age, were calculated. LMMs were used to determine the effect of LEQ scores on $\triangle$ age, adjusting for chronological age, sex, DNAm smoking scores, cell composition estimates, and technical batch effects (sample slide and array). Additionally, the difference in symptom ratings and stress scores between visits were calculated. The association between the change in symptoms and LEQ scores between baseline and 1-year follow-up with AA at 1-year follow-up was determined via linear regression models, again controlling for chronological age, sex, DNAm smoking scores, cell composition estimates and technical batch effects.

\section{Additional analyses}

Nominally significant CpGs (unadjusted $p<0.05$ ) associated with total LEQ scores were used for gene-based enrichment analysis using the GOmeth function from the Bioconductor package missMethyl. GOmeth maps a vector of $\mathrm{CpG}$ sites to Entrez Gene IDs, and tests for gene ontology (GO) term pathway enrichment using a hypergeometric test (Geeleher et al. 2013). Additionally, the correlation between DNAm in blood and four brain regions was explored for the most suggestive CpGs associated with total LEQ scores (see Additional file 1).

\section{Results}

\section{Change in methylation over time}

The mean absolute difference in methylation $(\beta)$ between visits 1 and $3(|\Delta \beta|)$ was calculated across all samples for all CpG sites (Fig. 1). Over the 1-year period, $|\Delta \beta|$ ranged from $<0.001$ to 0.299 with an average change of 0.014 . Of 753,251 CpG sites, only 68 had an $|\Delta \beta|$ of 0.10 or more, while 8454 sites differed by at least 0.05 between visits. Just over half of the sites $(428,610)$ showed an absolute difference in methylation of at least $1 \%$. Investigation of the functional genomic distribution of the least stable CpGs over time $(|\Delta \beta| \geq 0.10)$ revealed the majority of CpGs fell within Open Seas, while 12 fell within CpG Islands, and the remaining in CpG Shores and Shelves (Fig. 2). In summary, 34,776 CpG sites showed a nominally significant difference over time (unadjusted $p$-value $<0.05$ ), after correcting for age, sex, smoking and cell composition estimates. However, not a single locus withstood correction for multiple testing (FDR-corrected $p$-value $<0.05)$.

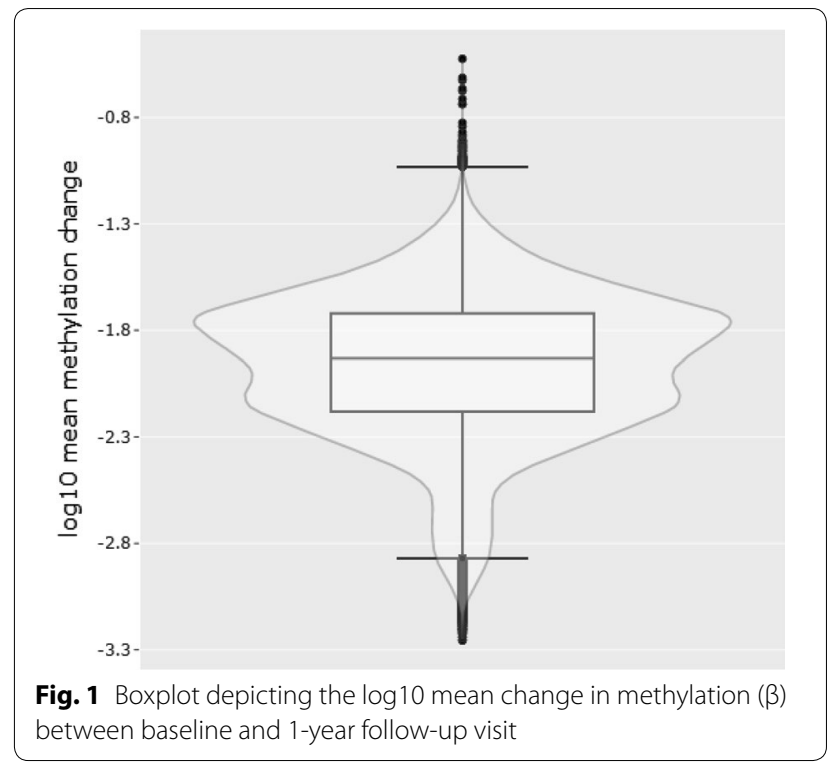




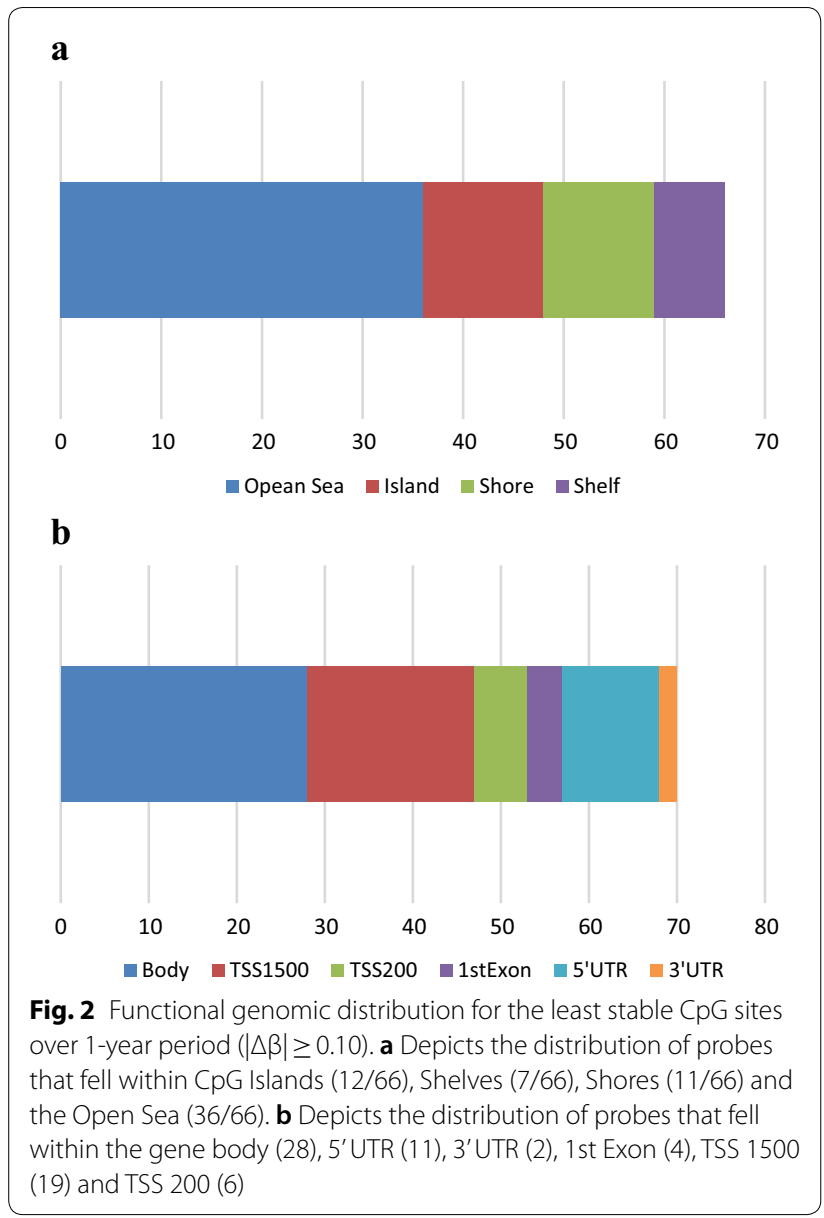

\section{Methylation association analysis}

We performed an exploratory analysis looking for associations between LEQ scores and DNAm in individual CpG probes in the vicinity of candidate genes previously implicated in the stress response and in the most variable $\mathrm{CpG}$ sites across the epigenome. Methylation at a single $\mathrm{CpG}$ site (cg15212455; POU6F2; "POU class 6 homeobox 2"; chr 7) was associated with impact ratings of total LEQ scores with a suggestive significance of $p<1.0 \times 10^{-5}$, although not a single locus withstood correction for multiple testing (FDR-corrected $p>0.05$ for all comparisons). Figure 3 shows the Manhattan plot depicting all analyzed CpG sites with their calculated $p$-values for the association between DNAm and total LEQ scores. Table 2 lists the top 20 loci associated at nominal significance with total LEQ scores. Inspection of quantile-quantile (QQ) plots did not show evidence for inflation or bias (Fig. 4; Lambda factor $=0.98$ ). Manhattan plots and associated QQ plots for additional association analyses can be found in Additional file 1: Fig. S3-S8. The sensitivity analysis, excluding subjects who did not take psychotropic drugs at the time of testing, did not yield signification

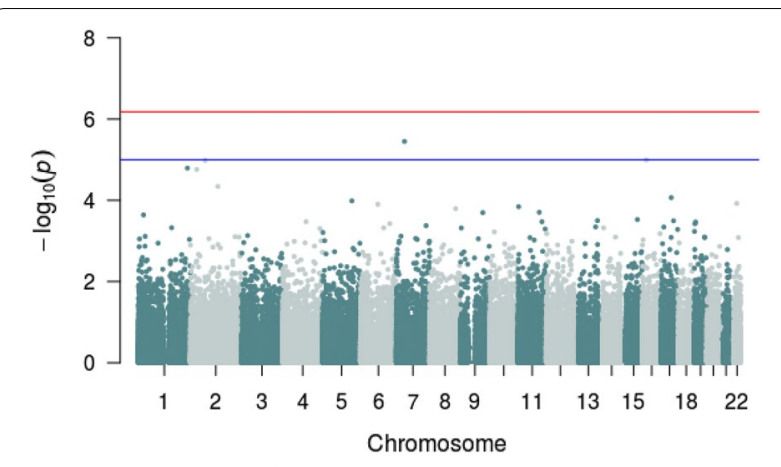

Fig. 3 Manhattan plot for association between DNA methylation and total LEQ scores. The horizontal red line represents the epigenome-wide significant threshold for this study $\left(p<6.6 \times 10^{-7}\right)$ and the blue line represents a suggestive significance threshold $\left(p<1.0 \times 10^{-5}\right)$

associations. These results, specific to modeling the association between DNAm and total LEQ scores, are presented in Additional file 1: Figs. S9 and S10.

\section{Epigenetic aging}

As expected, there was a strong positive correlation between individuals' DNAm age and chronological age $(r=0.941, p<0.001$; see Additional file 1: Fig. S11). According to Horvath's estimate, the mean (SD, range) AA was -0.23 years (3.71, range -9.94 to 9.86 years) at baseline and 0.25 years (3.95, range -8.12 to 9.43 years) at the 1-year follow-up. Between visits, the mean (SD, range) change in AA was 0.50 years (4.97, range -10.72 to 13.85 years). Overall, no statistically significant associations between epigenetic aging and symptom or stress measures were detected.

\section{Additional analyses}

We included genes mapped by the top CpG sites (unadjusted $p<0.05$ ) associated with total LEQ scores in an enrichment analysis. No biological processes survived FDR correction (see Additional file 1: Table S3). Blood brain correlation coefficients for methylation of the top 20 loci associated with total LEQ scores (overlapping with the $450 \mathrm{~K}$ Beadchip array) are presented in Table 3 . Eight of the top 20 most differentially methylated loci associated with total LEQ scores showed a significant correlation between methylation in the blood and methylation in at least one brain region. Methylation of the CpG site that was most strongly associated with total LEQ scores was significantly correlated with methylation in all four brain regions $(p<0.001$; see Additional file 1 : Fig. S12). 
Table 2 Top 20 Cpg sites associated with total LEQ scores

\begin{tabular}{|c|c|c|c|c|c|c|}
\hline Probe & $t$ value & $p$-value & $\begin{array}{l}\text { FDR-corrected } \\
p \text {-value }\end{array}$ & Chr & Relation to island & Annotated gene \\
\hline $\operatorname{cg} 15212455$ & -4.87 & $3.56 \mathrm{E}-06$ & 0.263 & chr7 & Open Sea & POU6F2 \\
\hline cg05335886 & -4.55 & $1.02 \mathrm{E}-05$ & 0.263 & chr16 & Island & TMC5 \\
\hline cg09725915 & 4.54 & $1.05 \mathrm{E}-05$ & 0.263 & chr2 & Island & \\
\hline cg24511004 & 4.43 & $1.62 \mathrm{E}-05$ & 0.263 & chr1 & Open Sea & \\
\hline $\operatorname{cg} 18110277$ & -4.50 & $1.74 \mathrm{E}-05$ & 0.263 & chr2 & Open Sea & \\
\hline cg21516302 & -4.24 & $4.58 \mathrm{E}-05$ & 0.575 & chr2 & Open Sea & \\
\hline cg05180443 & -4.04 & $8.61 \mathrm{E}-05$ & 0.927 & chr17 & Island & CHAD;ACSF2 \\
\hline cg01440452 & -3.97 & $1.03 \mathrm{E}-04$ & 0.946 & chr 5 & N Shore & PURA \\
\hline cg26730347 & -4.00 & $1.20 \mathrm{E}-04$ & 0.946 & chr22 & N Shore & SLC5A1 \\
\hline cg15869582 & 3.94 & $1.26 \mathrm{E}-04$ & 0.946 & chr6 & S Shore & IBTK \\
\hline cg05919744 & 3.95 & $1.44 \mathrm{E}-04$ & 0.977 & chr11 & S Shore & SLC22A18AS;SLC22A18 \\
\hline cg26822318 & 3.86 & $1.61 E-04$ & 0.977 & chr8 & Open Sea & FER1L6 \\
\hline $\operatorname{cg} 27296293$ & -3.80 & $1.98 \mathrm{E}-04$ & 0.977 & chr11 & Island & RP11-748H22.1; TRPC6 \\
\hline cg06334363 & 3.82 & $2.01 \mathrm{E}-04$ & 0.977 & chr9 & S Shore & RP11-235C23.5; FKTN \\
\hline cg00356897 & -3.79 & $2.30 \mathrm{E}-04$ & 0.977 & chr1 & Open Sea & RP4-594/10.2 \\
\hline cg24795825 & 3.72 & $2.98 \mathrm{E}-04$ & 0.977 & chr15 & N Shore & MORF4L 1 \\
\hline cg17984201 & 3.69 & $3.17 \mathrm{E}-04$ & 0.977 & chr13 & Open Sea & \\
\hline $\operatorname{cg} 18002447$ & -3.67 & $3.20 E-04$ & 0.977 & chr17 & Island & \\
\hline cg07349208 & -3.66 & $3.36 \mathrm{E}-04$ & 0.977 & chr4 & Island & RP11-380D23.2 \\
\hline cg05705044 & 3.65 & $3.38 \mathrm{E}-04$ & 0.977 & chr11 & S Shore & RBM7 \\
\hline
\end{tabular}

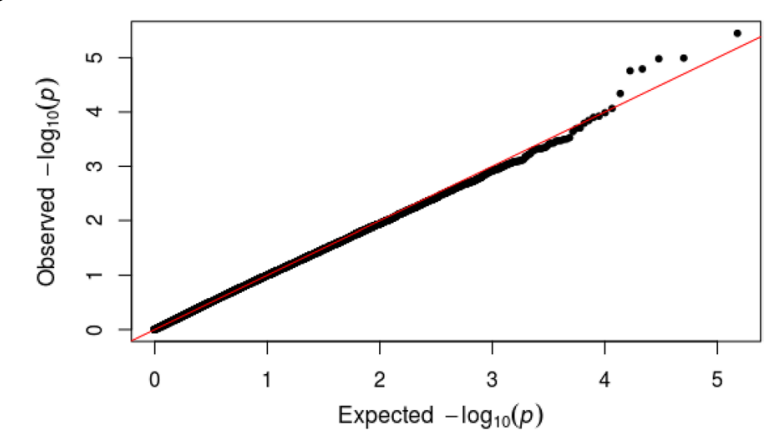

Fig. 4 QQ plot. The plot shows no evidence for inflation or bias in the association analysis of DNA methylation with total LEQ scores $($ Lambda $=1.04)$

\section{Discussion}

To the best of our knowledge, our study is the first to investigate epigenome-wide methylation changes over time in BD patients. Moreover, it is the first to explore methylation changes related to non-traumatic stressful life events on an epigenome-wide scale. Although no locus withstood correction for multiple testing, our suggestive findings and secondary analyses provide limited evidence supporting a role of DNAm in association with non-traumatic life events in chronic BD patients.
We identified a single, suggestively significant, CpG site associated with total LEQ scores, mapping to POU6F2, which has been associated with several psychiatric traits as well as intelligence and educational attainment. More specifically, genome-wide association studies have identified POU6F2 risk variants associated with psychological distress (Koshimizu et al. 2019), feeling emotionally hurt (Nagel et al. 2018), schizophrenia (Goes et al. 2015), autism (Anney et al. 2010), educational attainment (Lee et al. 2018; Okbay et al. 2016) and intelligence (Hill et al. 2018; Davies et al. 2018). Additionally, in a longitudinal investigation of DNAm changes preceding adolescent psychotic experiences, DNAm of the CpG site cg11604728 (POU6F2) measured at age 15-17 was among the top $20 \mathrm{CpG}$ sites indicative of psychotic experiences at age 18 (Roberts et al. 2019). Furthermore, POU6F2 is highly expressed in the brain with the highest expression found in the frontal cortex (Additional file 1: Fig. S13) and methylation of our suggestive CpG site in blood is correlated with methylation in brain tissue across multiple brain regions. Interestingly, another of our top $20 \mathrm{CpG}$ sites (cg26822318) falls in proximity to the FER1L6 gene, of which a variant (rs4870888) has been associated with suicide attempts in a meta-analysis of major depressive disorder, schizophrenia and BD (Mullins et al. 2019). Furthermore, another GWAS reported a FER1L6 
Table 3 Blood-brain methylation correlation for top differentially methylated CpGs associated with total LEQ scores

\begin{tabular}{|c|c|c|c|c|c|c|c|c|}
\hline Probe & Blood-PFC & $p$-value & Blood-EC & $p$-value & Blood-STG & $p$-value & Blood-CER & $p$-value \\
\hline cg15212455 & 0.721 & $4.16 E-13$ & 0.731 & $4.64 E-13$ & 0.747 & $1.48 E-14$ & 0.631 & 3.71E-09 \\
\hline cg05335886 & -0.086 & 0.467 & -0.101 & 0.404 & -0.145 & 0.213 & -0.155 & 0.196 \\
\hline cg09725915 & 0.576 & $7.86 E-08$ & 0.532 & $1.76 E-06$ & 0.626 & $1.96 E-09$ & 0.489 & $1.49 E-05$ \\
\hline cg21516302 & 0.373 & 0.001 & 0.522 & $3.01 E-06$ & 0.507 & $3.46 E-06$ & 0.307 & 0.009 \\
\hline cg05180443 & 0.204 & 0.081 & 0.336 & 0.004 & 0.298 & 0.009 & 0.175 & 0.144 \\
\hline cg01440452 & -0.097 & 0.413 & 0.062 & 0.606 & -0.119 & 0.309 & 0.020 & 0.870 \\
\hline $\operatorname{cg} 26730347$ & 0.499 & $6.05 E-06$ & 0.568 & $2.33 E-07$ & 0.562 & $1.51 E-07$ & 0.493 & $1.27 E-05$ \\
\hline cg27296293 & 0.131 & 0.265 & -0.214 & 0.073 & 0.037 & 0.755 & 0.236 & 0.048 \\
\hline $\operatorname{cg} 24795825$ & 0.016 & 0.894 & 0.299 & 0.011 & 0.247 & 0.033 & 0.121 & 0.317 \\
\hline cg18002447 & 0.038 & 0.749 & 0.034 & 0.776 & 0.042 & 0.720 & -0.262 & 0.028 \\
\hline cg07349208 & 0.167 & 0.156 & 0.095 & 0.431 & -0.112 & 0.338 & 0.068 & 0.571 \\
\hline
\end{tabular}

PFC prefrontal cortex, EC entorhinal cortex, STG superior temporal gyrus, CER cerebellum

Significant correlations in italics

variant (rs10481151) suggestively associated with cognitive performance (Need et al. 2009).

At the current sample size, our study provides only minimal evidence supporting an association between methylation of individual CpGs and non-traumatic, recent stressful life events in $\mathrm{BD}$. These findings, however, corroborate other reports of a limited role of DNAm with non-traumatic stress (Marzi et al. 2018). Noteworthy, a recent study reported hypermethylation of KITLG associated with childhood trauma in healthy controls $(n=91)$ but not in bipolar patients $(n=50)$ (He et al. 2018). Although the mechanistic role of DNAm in the phenotypic expression of early life adversities is well established in the literature, other mechanisms may be responsible in adulthood and in association with subsequent events. This notion aligns with theories such as Post's kindling hypothesis and the decay model which suggest a higher impact of life events on first episode than on subsequent episodes in BD (Aldinger and Schulze 2017; Kemner et al. 2015; Hillegers et al. 2004). Furthermore, it must be considered whether positive epigenetic associations with life events could be disorder-specific, genotype-dependent, associated with specific trauma exposure, age groups, sex and/or tissues measured (Marzi et al. 2018; Vinkers et al. 2015; Uddin et al. 2010; Boks et al. 2015; Smith et al. 2011; Mehta et al. 2017). While there is no gold standard for life stress measurements, differences in how to quantify stress may also have a major effect on findings (Johnson 2005; Bender and Alloy 2011; Monroe 2008; Dohrenwend 2010; Brown and Harris 2012).

The main strength of our study is its longitudinal design, allowing for repeated measures within individuals and to investigate methylation changes over time and in relation to symptomatology and stressful life events. To the best of our knowledge, this is the first study to collect repeated epigenome-wide methylation measures in bipolar patients. Furthermore, our study paid attention to critical confounding factors which often lead to spurious findings. For example, the use of methylation-based smoking scores better controls for the extent of smoking throughout the lifetime than the use of self-reported smoking measures (Elliott et al. 2014; Shenker et al. 2013). Finally, in contrast to most other studies, we have included an exploratory epigenome-wide approach.

Despite the strengths of our study, several limitations need to be addressed. First, our study was limited by our small sample size which makes identifying subtle differences in methylation difficult. Taking power into consideration, and as an attempt to address the inherent multiple testing problem associated with EWAS, we limited our EWAS to only the most variable CpG sites according to MAD scores. While the fact that not a single site-specific association in DNAm survived correction for multiple testing could reflect the limited statistical power of our small sample, it may also be related to an overly conservative multiple testing correction considering the lack of variability in methylation at many CpGs and spatial correlation of methylation with nearby sites (Walker et al. 2016; Lunnon et al. 2015). A recent study estimated there are approximately 530,000 independent tests in a whole blood EPIC array DNAm study. Accordingly, they proposed a corrected significance threshold of $9.42 \times 10^{-8}$ to be used as a standard threshold for future EWAS based on the EPIC array (Mansell et al. 2019). Furthermore, the study introduced a freely available online tool which allows users to perform power calculations to guide sample sizes, accounting for the individual properties of each DNAm site and using their empirically derived significance threshold. According to their tool, an effect size of just 
$1 \%$ difference between cases and controls would require a sample of 1000 participants, for only a third of methylation sites to have $>80 \%$ power. We observed an effect size below $5 \%$ in our study (based on median split) for our most significantly associated site, indicating that our study is nevertheless underpowered. Future studies should take advantage of this tool to assess, a priori, required sample sizes according to their expected effect sizes. Furthermore, complementary systems biology approaches such as weighted gene co-methylation network analysis (WGCNA) could be beneficial for studies with limited sample sizes, providing more insight into the functional role of altered DNAm (Langfelder and Horvath 2008).

Another limitation is in relation to the fact that our sample represents a cohort of chronic BD patients which likely influenced our investigation of epigenetic aging related to symptom ratings over time. The chronicity of patients may also confound our findings with regards to the heterogenous treatments patients have received over the years. To acknowledge this critical factor, we conducted a sensitivity analysis excluding those subjects not taking psychotropic drugs at the time of testing, however, this also did not lead to significant results. One must also consider the possible recall and desirability biases associated with self-rating questionnaires like the LEQ and CTS. Lastly, little is known about the temporal stability of epigenetic markers (Byun et al. 2012; Talens et al. 2010). We cannot be sure whether the time interval of 1 year was too long or short to observe dramatic methylation changes or at what time window following exposure to stressful life events one might observe changed methylation profiles.

\section{Conclusions}

$\mathrm{BD}$ is a multifactorial psychiatric illness, and for many patients full interepisodic remission never occurs (Sam et al. 2019). Stressful life events have been associated with a worse course of BD (Aldinger and Schulze 2017) and there remains a need to better understand the mechanisms which allow these stressors to bring about phenotypic change. Our study provides limited evidence supporting an association between DNAm and recent, non-traumatic stressful life events in $\mathrm{BD}$ patients. As findings in clinical populations have been inconsistent, there is still much to be understood especially with regards to the temporal nature of environmentally induced DNA modifications. Future larger studies of well-characterized patients, longitudinal in design, are warranted.

\section{Supplementary information}

Supplementary information accompanies this paper at https://doi. org/10.1186/s40345-019-0176-6.

Additional file 1. Supporting methods, Figures S1-S13, and Tables S1-S3.

\section{Abbreviations}

BD: bipolar disorder; GWAS: genome-wide association studies; DNAm: DNA methylation; CpG: cytosine-guanine dinucleotides; HPA: hypothalamic-pituitary-adrenal; LEQ: Life Events Questionnaire; CTS: Childhood Trauma Screener; PANSS: Positive and Negative Syndrome Scale; GAF: Global Assessment of Functioning; YMRS: Young Mania Rating Scale; IDS- $C_{30}$ : Inventory of Depressive Symptomatology; FunNorm: functional normalization; EWAS: epigenomewide association analysis; BMIQ: beta-mixture quantile dilation; LMM: linear mixed-effects model; FDR: false discovery rate; MAD: median absolute deviation; AA: age acceleration; GO: gene ontology; QQ: quantile-quantile; WGCNA: weighted gene co-methylation network analysis.

\section{Acknowledgements}

The Genotype-Tissue Expression (GTEx) Project was supported by the Common Fund of the Office of the Director of the National Institutes of Health, and by NCl, NHGRI, NHLBI, NIDA, NIMH, and NINDS. The data used for the gene tissue expression analysis described in the this manuscript and reported in Additional file 1 were obtained from the GTEx portal: GTEx Analysis Release V8 (dbGaP Accession phs000424.v8.p2) on 09/24/19.

\section{Authors' contributions}

All authors made substantial contributions to the conception or design of the work, or the acquisition, analysis or interpretation of data. Authors ALC and TGS designed the study. Authors TGS and UH provided supervision. Author ALC conducted the analysis in consultation with UH, SS, DC and TA. Author ALC wrote the first draft of the manuscript. All authors contributed to the manuscript. All authors read and approved the final manuscript.

\section{Funding}

Thomas G. Schulze and Peter Falkai are supported by the German Research Foundation (Deutsche Forschungsgemeinschaft; DFG) within the framework of the projects http://www.kfo241.de and http://www.PsyCourse.de (SCHU 1603/4-1, 5-1, 7-1; FA241/16-1). The genotyping was in part funded by the German Federal Ministry of Education and Research (BMBF) through the Integrated Network IntegraMent (Integrated Understanding of Causes and Mechanisms in Mental Disorders), under the auspices of the e:Med Program with grants awarded to Thomas G. Schulze (01ZX1614K), Marcella Rietschel (01ZX1614G), and Markus M. Nöthen (01ZX1614A). Thomas G. Schulze received additional support from the German Federal Ministry of Education and Research (BMBF) within the framework of the BipoLife network (01EE1404H) and the Dr. Lisa Oehler Foundation (Kassel, Germany). Sergi Papiol was supported by a 2016 NARSAD Young Investigator Grant (25015) from the Brain and Behavior Research Foundation. Marcella Rietschel and Markus M. Nöthen received funding from the German Research Foundation (DFG, grant FOR2107; RI 908/11-2 to Marcella Rietschel, NO 246/10-2 to Markus M. Nöthen). Franziska Degenhardt received support from the BONFOR Programme of the University of Bonn, Germany. Jens Wiltfang is supported by an llídio Pinho professorship and BBiMED (UID/BIM/04501/2013), at the University of Aveiro.

\section{Availability of data and materials}

Participants of the PsyCourse study have consented to the sharing of their pseudonymized data with other researchers and research consortia. Thus, PsyCourse data will be made available to bona fide researchers collaborating with us given a mutually agreed written memorandum of understanding has been signed.

\section{Ethics approval and consent to participate}

The study was approved by the local ethics committee for each study center and was carried out following the rules of the Declaration of Helsinki. 


\section{Consent for publication}

Not applicable.

\section{Competing interests}

Dr. Jens Reimer received honoraria from Otsuka-Lundbeck for participation in a speakers' bureau. Dr. Max Schmauß received honoraria form OtsukaLundbeck, Neuraxpharm, Aristo and Janssen for participation in the speakers's bureau or advisory boards. All other authors declare that they have no competing interests.

\section{Author details}

${ }^{1}$ Institute of Psychiatric Phenomics and Genomics, University Hospital, LMU Munich, Nussbaumstrasse 7, 80336 Munich, Germany. ${ }^{2}$ International Max Planck Research School for Translational Psychiatry (IMPRS-TP), 80804 Munich, Germany. ${ }^{3}$ Department of Translational Research in Psychiatry, Max Planck Institute of Psychiatry, 80804 Munich, Germany. ${ }^{4}$ Department of Psychiatry and Psychotherapy, University Hospital, LMU Munich, 80336 Munich, Germany. ${ }^{5}$ Department of Psychiatry and Psychotherapy, University Medical Center Göttingen, 37075 Göttingen, Germany. ${ }^{6}$ Department of Neurology, Klinikum rechts der Isar, School of Medicine, Technical University of Munich, 81675 Munich, Germany. ${ }^{7}$ Department of Psychiatry, Ruhr University Bochum, LWL University Hospital, 44791 Bochum, Germany. ${ }^{8}$ Department of Psychiatry and Psychotherapy, Bezirkskrankenhaus Augsburg, University of Augsburg, 86156 Augsburg, Germany. ${ }^{9}$ Psychiatrieverbund Oldenburger Land gGmbH, Karl-Jaspers-Klinik, 26160 Bad Zwischenahn, Germany. ${ }^{10}$ Department of Psychiatry and Psychotherapy, University Medical Center Hamburg-Eppendorf, 20246 Hamburg, Germany. ${ }^{11}$ Department of Psychiatry and Psychotherapeutic Medicine, Research Unit for Bipolar Affective Disorder, Medical University of Graz, $8036 \mathrm{Graz}$, Austria. ${ }^{12}$ Department of Psychiatry, Dr. Frontheim-Mental Health, 38704 Liebenburg, Germany. ${ }^{13}$ Department of Psychiatry and Psychotherapy, Agaplesion Diakonieklinikum, 27356 Rotenburg, Germany. ${ }^{14}$ Karl-Jaspers Clinic, European Medical School Oldenburg-Groningen, 26160 Oldenburg, Germany. ${ }^{15}$ Clinic for Psychiatry and Psychotherapy, Clinical Center Werra-Meißner, 37269 Eschwege, Germany. ${ }^{16}$ Asklepios Specialized Hospital, 37081 Göttingen, Germany. ${ }^{17}$ AMEOS Clinical Center Hildesheim, 31135 Hildesheim, Germany. ${ }^{18}$ Center für Systems Neuroscience (ZSN) Hannover, 30559 Hannover, Germany. ${ }^{19}$ Department of Psychiatry, Medical School of Hannover, 30625 Hannover, Germany. ${ }^{20}$ Psychiatric Hospital Lüneburg, 21339 Lüneburg, Germany. ${ }^{21}$ AMEOS Clinical Center Osnabrück, 49088 Osnabrück, Germany. ${ }^{22}$ Department of Genetic Epidemiology in Psychiatry, Central Institute of Mental Health, Medical Faculty Mannheim, University of Heidelberg, 68159 Mannheim, Germany. ${ }^{23}$ Institute of Human Genetics, University of Bonn, School of Medicine \& University Hospital Bonn, 53127 Bonn, Germany. ${ }^{24}$ Center for Human Genetics, University of Marburg, 35033 Marburg, Germany. ${ }^{25}$ Department of Biomedicine, University of Basel, 4031 Basel, Switzerland. ${ }^{26}$ Department of Psychiatry (UPK), University of Basel, 4002 Basel, Switzerland. ${ }^{27}$ German Center for Neurodegenerative Diseases (DZNE), 37075 Göttingen, Germany. ${ }^{28}$ iBiMED, Medical Sciences Department, University of Aveiro, 3810-193 Aveiro, Portugal.

Received: 25 September 2019 Accepted: 18 December 2019 Published online: 12 February 2020

\section{References}

Aas M, Henry C, Andreassen OA, Bellivier F, Melle I, Etain B. The role of childhood trauma in bipolar disorders. Int J Bipolar Disord. 2016;4:2.

Alasaari JS, Lagus M, Ollila HM, Toivola A, Kivimäki M, Vahtera J, et al. Environmental stress affects DNA methylation of a $\mathrm{CpG}$ rich promoter region of serotonin transporter gene in a nurse cohort. PLOS ONE. 2012;7(9):e45813.

Aldinger F, Schulze TG. Environmental factors, life events, and trauma in the course of bipolar disorder. Psychiatry Clin Neurosci. 2017;71 (1):6-17.

Alloy LB, Abramson LY, Urosevic S, Walshaw PD, Nusslock R, Neeren AM. The psychosocial context of bipolar disorder: environmental, cognitive, and developmental risk factors. Clin Psychol Rev. 2005;25(8):1043-75.

American Psychiatric Association. Diagnostic and statistical manual of mental disorders. 4th ed. Washington, DC: American Psychiatric Association; 2002.
Anney R, Klei L, Pinto D, Regan R, Conroy J, Magalhaes TR, et al. A genomewide scan for common alleles affecting risk for autism. Hum Mol Genet. 2010;19(20):4072-82.

Aryee MJ, Jaffe AE, Corrada-Bravo H, Ladd-Acosta C, Feinberg AP, Hansen KD, et al. Minfi: a flexible and comprehensive bioconductor package for the analysis of infinium DNA methylation microarrays. Bioinformatics. 2014;30(10):1363-9.

Bates D, Maechler M, Bolker B, Walker S. Fitting linear mixed-effects models using Ime4. J Stat Softw. 2015;67(1):1-48.

Bender RE, Alloy LB. Life stress and kindling in bipolar disorder: review of the evidence and integration with emerging biopsychosocial theories. Clin Psychol Rev. 2011;31(3):383-98.

Benjamini $Y$, Hochberg $Y$. Controlling the false discovery rate: a practical and powerful approach to multiple testing. J R Stat Soc B. 1995;57(1):289-300.

Bernstein DP, Ahluvalia T, Pogge D, Handelsman L. Validity of the Childhood Trauma Questionnaire in an adolescent psychiatric population. J Am Acad Child Adolesc Psychiatry. 1997;36(3):340-8.

Bernstein DP, Stein JA, Newcomb MD, Walker E, Pogge D, Ahluvalia T, et al. Development and validation of a brief screening version of the Childhood Trauma Questionnaire. Child Abuse Negl. 2003;27(2):169-90.

Boks MP, van Mierlo HC, Rutten BP, Radstake TR, De Witte L, Geuze E, et al. Longitudinal changes of telomere length and epigenetic age related to traumatic stress and post-traumatic stress disorder. Psychoneuroendocrinology. 2015;51:506-12.

Brown GW, Harris T. Social origins of depression: a study of psychiatric disorder in women. New York: Routledge; 2012.

Budde M, Anderson-Schmidt H, Gade K, Reich-Erkelenz D, Adorjan K, Kalman $J \mathrm{~L}$, et al. A longitudinal approach to biological psychiatric research: the PsyCourse study. Am J Med Genet B Neuropsychiatr Genet. 2019;180(2):89-102

Byun HM, Nordio F, Coull BA, Tarantini L, Hou L, Bonzini M, et al. Temporal stability of epigenetic markers: sequence characteristics and predictors of short-term DNA methylation variations. PLoS ONE. 2012;7(6):e39220.

Chen YA, Lemire M, Choufani S, Butcher DT, Grafodatskaya D, Zanke BW, et al. Discovery of cross-reactive probes and polymorphic CpGs in the Illumina Infinium HumanMethylation450 microarray. Epigenetics. 2013;8(2):203-9.

Davies G, Lam M, Harris SE, Trampush JW, Luciano M, Hill WD, et al. Study of 300,486 individuals identifies 148 independent genetic loci influencing general cognitive function. Nat Commun. 2018;9(1):2098.

Dohrenwend BP. Toward a typology of high-risk major stressful events and situations in posttraumatic stress disorder and related psychopathology. Psychol Inj Law. 2010;3(2):89-99.

Duffy A, Goodday SM, Keown-Stoneman C, Scotti M, Maitra M, Nagy C, et al. Epigenetic markers in inflammation-related genes associated with mood disorder: a cross-sectional and longitudinal study in high-risk offspring of bipolar parents. Int J Bipolar Disord. 2019;7(1):17.

Duman EA, Canli T. Influence of life stress, 5-HTTLPR genotype, and SLC6A4 methylation on gene expression and stress response in healthy Caucasian males. Biol Mood Anxiety Disord. 2015;5:2.

Elliott HR, Tillin T, McArdle WL, Ho K, Duggirala A, Frayling TM, et al. Differences in smoking associated DNA methylation patterns in South Asians and Europeans. Clin Epigenet. 2014;6(1):4.

Endicott J, Spitzer RL, Fleiss JL, Cohen J. The Global Assessment Scale: a procedure for measuring overall severity of psychiatric disturbance. Arch Gen Psychiatry. 1976;33(6):766-71.

Fortin J-P, Labbe A, Lemire M, Zanke BW, Hudson TJ, Fertig EJ, et al. Functional normalization of $450 \mathrm{k}$ methylation array data improves replication in large cancer studies. Genome Biol. 2014;15(11):503.

Fries GR, Li Q, McAlpin B, Rein T, Walss-Bass C, Soares JC, et al. The role of DNA methylation in the pathophysiology and treatment of bipolar disorder. Neurosci Biobehav Rev. 2016;68:474-88.

Geeleher P, Hartnett L, Egan LJ, Golden A, Raja Ali RA, Seoighe C. Gene-set analysis is severely biased when applied to genome-wide methylation data. Bioinformatics. 2013;29(15):1851-7.

Glaesmer H, Schulz A, Hauser W, Freyberger HJ, Brahler E, Grabe HJ. The childhood trauma screener (CTS) - development and validation of cut-offscores for classificatory diagnostics. Psychiatr Prax. 2013;40(4):220-6.

Goes FS, McGrath J, Avramopoulos D, Wolyniec P, Pirooznia M, Ruczinski I, et al. Genome-wide association study of schizophrenia in Ashkenazi Jews. Am J Med Genet Part B Neuropsychiatr Genet. 2015;168(8):649-59. 
Goodwin FK, Jamison KR. Manic-depressive illness: bipolar disorders and recurrent depression. 2nd ed. New York: Oxford University Press; 2007.

Grabe HJ, Schulz A, Schmidt CO, Appel K, Driessen M, Wingenfeld K, et al. Ein Screeninginstrument für Missbrauch und Vernachlässigung in der Kindheit: der Childhood Trauma Screener (CTS). Psychiatr Prax. 2012;39(03):109-15.

He Y, Vinkers CH, Houtepen LC, de Witte LD, Boks MP. Childhood adversity is associated with increased KITLG methylation in healthy individuals but not in bipolar disorder patients. Front Psychiatry. 2018;9:743.

Hill WD, Marioni RE, Maghzian O, Ritchie SJ, Hagenaars SP, McIntosh AM, et al. A combined analysis of genetically correlated traits identifies 187 loci and a role for neurogenesis and myelination in intelligence. Mol Psychiatry. 2018:24(2):169-81.

Hillegers MH, Burger H, Wals M, Reichart CG, Verhulst FC, Nolen WA, et al. Impact of stressful life events, familial loading and their interaction on the onset of mood disorders: study in a high-risk cohort of adolescent offspring of parents with bipolar disorder. Br J Psychiatry. 2004;185:97-101.

Horvath S. DNA methylation age of human tissues and cell types. Genome Biol. 2013;14(10):R115.

Houseman EA, Accomando WP, Koestler DC, Christensen BC, Marsit CJ, Nelson $\mathrm{HH}$, et al. DNA methylation arrays as surrogate measures of cell mixture distribution. BMC Bioinform. 2012;13:86.

Jaworska-Andryszewska P, Rybakowski JK. Childhood trauma in mood disorders: neurobiological mechanisms and implications for treatment. Pharmacol Rep. 2019;71(1):112-20.

Johnson SL. Life events in bipolar disorder: towards more specific models. Clin Psychol Rev. 2005;25(8):1008-27.

Johnson WE, Li C, Rabinovic A. Adjusting batch effects in microarray expression data using empirical Bayes methods. Biostatistics. 2007;8(1):118-27.

Kay SR, Fiszbein A, Opler LA. The positive and negative syndrome scale (PANSS) for schizophrenia. Schizophr Bull. 1987:13(2):261-76.

Kemner SM, van Haren NE, Bootsman F, Eijkemans MJ, Vonk R, van der Schot $A C$, et al. The influence of life events on first and recurrent admissions in bipolar disorder. Int J Bipolar Disord. 2015;3:6.

Kobeissy F, Alawieh A, Mondello S, Boustany RM, Gold MS. Biomarkers in psychiatry: how close are we? Front Psychiatry. 2012;3:114.

Koshimizu H, Nogawa S, Asano S, Ikeda M, Iwata N, Takahashi S, et al. Genomewide association study identifies a novel locus associated with psychological distress in the Japanese population. Transl Psychiatry. 2019;9(1):52.

Kular L, Kular S. Epigenetics applied to psychiatry: clinical opportunities and future challenges. Psychiatry Clin Neurosci. 2018;72(4):195-211.

Langfelder P, Horvath S. WGCNA: an R package for weighted correlation network analysis. BMC Bioinform. 2008;9(1):559.

Lee KWK, Pausova Z. Cigarette smoking and DNA methylation. Front Genet. 2013;4:132

Lee JJ, Wedow R, Okbay A, Kong E, Maghzian O, Zacher M, et al. Gene discovery and polygenic prediction from a genome-wide association study of educational attainment in 1.1 million individuals. Nat Genet. 2018;50(8):1112-21.

Lex C, Bazner E, Meyer TD. Does stress play a significant role in bipolar disorder? A meta-analysis. J Affect Disord. 2017;208:298-308,

Li Y, Camarillo C, XU J, Arana TB, Xiao Y, Zhao Z, et al. Genome-wide methylome analyses reveal novel epigenetic regulation patterns in schizophrenia and bipolar disorder. Biomed Res Int. 2015;2015:201587.

Luborsky L. Clinicians'judgments of mental health: a proposed scale. Arch Gen Psychiatry. 1962;7(6):407-17.

Ludwig B, Dwivedi Y. Dissecting bipolar disorder complexity through epigenomic approach. Mol Psychiatry. 2016;21 (11):1490-8.

Lunnon K, Smith RG, Cooper I, Greenbaum L, Mill J, Beeri MS. Blood methylomic signatures of presymptomatic dementia in elderly subjects with type 2 diabetes mellitus. Neurobiol Aging. 2015;36(3):1600.e1-e4.

Mansell G, Gorrie-Stone TJ, Bao Y, Kumari M, Schalkwyk LS, Mill J, et al. Guidance for DNA methylation studies: statistical insights from the Illumina EPIC array. BMC Genomics. 2019;20(1):366

Marzi SJ, Sugden K, Arseneault L, Belsky DW, Burrage J, Corcoran DL, et al. Analysis of DNA methylation in young people: limited evidence for an association between victimization stress and epigenetic variation in blood. Am J Psychiatry. 2018;175(6):517-29.

Matosin N, Cruceanu C, Binder EB. Preclinical and clinical evidence of DNA methylation changes in response to trauma and chronic stress. Chronic Stress. 2017. https://doi.org/10.1177/2470547017710764.
McGowan PO, Sasaki A, D’Alessio AC, Dymov S, Labonte B, Szyf M, et al. Epigenetic regulation of the glucocorticoid receptor in human brain associates with childhood abuse. Nat Neurosci. 2009;12(3):342-8.

Meana $\mathrm{JJ}$, Mollinedo-Gajate I. Biomarkers in psychiatry: between myth and clinical reality. Revista de Psiquiatría y Salud Mental. 2017;10(4):183-4 (English Edition).

Mehta D, Bruenig D, Carrillo-Roa T, Lawford B, Harvey W, Morris CP, et al. Genomewide DNA methylation analysis in combat veterans reveals a novel locus for PTSD. Acta Psychiatr Scand. 2017;136(5):493-505.

Monk C, Spicer J, Champagne FA. Linking prenatal maternal adversity to developmental outcomes in infants: the role of epigenetic pathways. Dev Psychopathol. 2012;24(4):1361-76.

Monroe SM. Modern approaches to conceptualizing and measuring human life stress. Annu Rev Clin Psychol. 2008:4:33-52.

Mullins N, Bigdeli TB, Borglum AD, Coleman JRI, Demontis D, Mehta D, et al. GWAS of suicide attempt in psychiatric disorders and association with major depression polygenic risk scores. Am J Psychiatry. 2019:176(8):651-60

Myaki K, Suzuki T, Song Y, Tsutsumi A, Kawakami N, Takahashi M, et al. Epigenetic changes caused by occupational stress in humans revealed through noninvasive assessment of DNA methylation of the tyrosine hydroxylase gene. J Neurol Neurol Disord. 2015;2(2):201.

Nagel M, Watanabe K, Stringer S, Posthuma D, van der Sluis S. Item-level analyses reveal genetic heterogeneity in neuroticism. Nat Commun. 2018;9(1):905.

Need AC, Attix DK, McEvoy JM, Cirulli ET, Linney KL, Hunt P, et al. A genomewide study of common SNPs and CNVs in cognitive performance in the CANTAB. Hum Mol Genet. 2009;18(23):4650-61.

Norbeck JS. Modification of life event questionnaires for use with female respondents. Res Nurs Health. 1984;7(1):61-71.

Okbay A, Beauchamp JP, Fontana MA, Lee JJ, Pers TH, Rietveld CA, et al. Genome-wide association study identifies 74 loci associated with educational attainment. Nature. 2016;533(7604):539-42.

Paykel ES. Life events and affective disorders. Acta Psychiatr Scand. 2003;108(s418):61-6.

Pishva E, Kenis G, van den Hove D, Lesch KP, Boks MP, van Os J, et al. The epigenome and postnatal environmental influences in psychotic disorders. Soc Psychiatry Psychiatr Epidemiol. 2014;49(3):337-48.

R Core Team. R: a language and environment for statistical computing. Vienna: R Foundation for Statistical Computing; 2014. https://www.R-project.org/.

Roberts S, Suderman M, Zammit S, Watkins SH, Hannon E, Mill J, et al. Longitudinal investigation of DNA methylation changes preceding adolescent psychotic experiences. Transl Psychiatry. 2019;9(1):69.

Rowland TA, Marwaha S. Epidemiology and risk factors for bipolar disorder. Ther Adv Psychopharmacol. 2018;8(9):251-69.

Sam SP, Nisha A, Varghese PJ. Stressful life events and relapse in bipolar affective disorder: a cross-sectional study from a tertiary care center of southern India. Indian J Psychol Med. 2019;41 (1):61-7.

Sarason IG, Johnson JH, Siegel JM. Assessing the impact of life changes: development of the Life Experiences Survey. J Consult Clin Psychol. 1978:46(5):932-46.

Sharma S, Powers A, Bradley B, Ressler KJ. Gene x environment determinants of stress- and anxiety-related disorders. Annu Rev Psychol. 2016;67:239-61.

Shenker NS, Polidoro S, van Veldhoven K, Sacerdote C, Ricceri F, Birrell MA, et al. Epigenome-wide association study in the European Prospective Investigation into Cancer and Nutrition (EPIC-Turin) identifies novel genetic loci associated with smoking. Hum Mol Genet. 2013;22(5):843-51.

Smith AK, Conneely KN, Kilaru V, Mercer KB, Weiss TE, Bradley B, et al. Differential immune system DNA methylation and cytokine regulation in post-traumatic stress disorder. Am J Med Genet B Neuropsychiatr Genet. 2011;156b(6):700-8.

Song Y, Miyaki K, Suzuki T, Sasaki Y, Tsutsumi A, Kawakami N, et al. Altered DNA methylation status of human brain derived neurotrophis factor gene could be useful as biomarker of depression. Am J Med Genet B Neuropsychiatr Genet. 2014;165b(4):357-64.

Stahl EA, Breen G, Forstner AJ, McQuillin A, Ripke S, Trubetskoy V, et al. Genome-wide association study identifies 30 loci associated with bipolar disorder. Nat Genet. 2019;51(5):793-803. 
Talens RP, Boomsma DI, Tobi EW, Kremer D, Jukema JW, Willemsen G, et al. Variation, patterns, and temporal stability of DNA methylation: considerations for epigenetic epidemiology. FASEB J. 2010;24(9):3135-44.

Teschendorff AE, Marabita F, Lechner M, Bartlett T, Tegner J, Gomez-Cabrero D, et al. A beta-mixture quantile normalization method for correcting probe design bias in Illumina Infinium 450k DNA methylation data. Bioinformatics. 2013;29(2):189-96.

Trivedi MH, Rush AJ, Ibrahim HM, Carmody TJ, Biggs MM, Suppes T, et al. The Inventory of Depressive Symptomatology, Clinician Rating (IDS-C) and Self-Report (IDS-SR), and the Quick Inventory of Depressive Symptomatology, Clinician Rating (QIDS-C) and Self-Report (QIDS-SR) in public sector patients with mood disorders: a psychometric evaluation. Psychol Med. 2004;34(1):73-82.

Uddin M, Aiello AE, Wildman DE, Koenen KC, Pawelec G, de Los Santos R, et al. Epigenetic and immune function profiles associated with posttraumatic stress disorder. Proc Natl Acad Sci USA. 2010;107(20):9470-5.

Vinkers CH, Kalafateli AL, Rutten BP, Kas MJ, Kaminsky Z, Turner JD, et al. Traumatic stress and human DNA methylation: a critical review. Epigenomics. 2015;7(4):593-608.
Walker RM, Sussmann JE, Whalley HC, Ryan NM, Porteous DJ, Mclntosh AM, et al. Preliminary assessment of pre-morbid DNA methylation in individuals at high genetic risk of mood disorders. Bipolar Disord. 2016;18(5):410-22.

Weaver IC, Cervoni N, Champagne FA, D'Alessio AC, Sharma S, Seckl JR, et al. Epigenetic programming by maternal behavior. Nat Neurosci. 2004;7(8):847-54

Young RC, Biggs JT, Ziegler VE, Meyer DA. A rating scale for mania: reliability, validity and sensitivity. Br J Psychiatry. 1978;133:429-35.

Zeilinger S, Kühnel B, Klopp N, Baurecht H, Kleinschmidt A, Gieger C, et al. Tobacco smoking leads to extensive genome-wide changes in DNA methylation. PLOS ONE. 2013;8(5):e63812.

\section{Publisher's Note}

Springer Nature remains neutral with regard to jurisdictional claims in published maps and institutional affiliations.

\section{Submit your manuscript to a SpringerOpen ${ }^{\circ}$ journal and benefit from:}

- Convenient online submission

- Rigorous peer review

- Open access: articles freely available online

- High visibility within the field

- Retaining the copyright to your article

Submit your next manuscript at $\boldsymbol{\nabla}$ springeropen.com 